\title{
Dopaminergic involvement during mental fatigue in health and cocaine addiction
}

\author{
SJ Moeller ${ }^{1}$, D Tomasi ${ }^{2}$, J Honorio ${ }^{1,3}$, ND Volkow ${ }^{2,4}$ and RZ Goldstein ${ }^{1}$
}

Dopamine modulates executive function, including sustaining cognitive control during mental fatigue. Using event-related functional magnetic resonance imaging (fMRI) during the color-word Stroop task, we aimed to model mental fatigue with repeated task exposures in 33 cocaine abusers and 20 healthy controls. During such mental fatigue (indicated by increased errors, and decreased post-error slowing and dorsal anterior cingulate response to error as a function of time-on-task), healthy individuals showed increased activity in the dopaminergic midbrain to error. Cocaine abusers, characterized by disrupted dopamine neurotransmission, showed an opposite pattern of response. This midbrain fMRI activity with repetition was further correlated with objective indices of endogenous motivation in all subjects: a state measure (task reaction time) and a trait measure (dopamine D2 receptor availability in caudate, as revealed by positron emission tomography data collected in a subset of this sample, which directly points to a contribution of dopamine to these results). In a second sample of 14 cocaine abusers and 15 controls, administration of an indirect dopamine agonist, methylphenidate, reversed these midbrain responses in both groups, possibly indicating normalization of response in cocaine abusers because of restoration of dopamine signaling but degradation of response in healthy controls owing to excessive dopamine signaling. Together, these multimodal imaging findings suggest a novel involvement of the dopaminergic midbrain in sustaining motivation during fatigue. This region might provide a useful target for strengthening self-control and/or endogenous motivation in addiction.

Translational Psychiatry (2012) 2, e176; doi:10.1038/tp.2012.110; published online 23 October 2012

\section{Introduction}

Drug addiction is characterized by functional and structural impairments in prefrontal cortical regions subserving selfregulation and top-down executive control, ${ }^{1}$ culminating in disadvantageous/impulsive decision-making, ${ }^{2}$ dysregulated inhibitory control, ${ }^{3}$ and poor behavioral adaptation. ${ }^{4}$ As these executive functions are subject to fatigue after their sustained use even in healthy individuals (reviewed in Heatherton and Wagner ${ }^{5}$ ), studying mental fatigue and its relation to selfcontrol could be especially important in a substance-abusing population. Supporting this suggestion, on days underage drinkers experienced higher-than-average self-control demands (which putatively resulted in mental fatigue), they were more likely to drink heavily-particularly if they initially had strong intentions to avoid overindulging. ${ }^{6}$ The reciprocal relationship has also received support: resisting the temptation to drink alcohol (again putatively resulting in mental fatigue) thwarted self-control on subsequent tasks of executive function. ${ }^{7}$ Further supporting the importance of mental fatigue in addiction are studies of fatigue/sleep deprivation. During early abstinence, poor sleep quality in cocaineaddicted individuals degrades neuropsychological functioning (reviewed in Morgan and Malison ${ }^{8}$ ), which in turn has been shown to impact treatment retention. ${ }^{9,10}$ In cigarette smokers, sleep deprivation increased the number of cigarettes subjects chose to smoke in a laboratory choice procedure (cigarettes versus money). ${ }^{11}$ Female smokers who had relapsed at 3-month follow-up reported more drowsiness than did those who maintained abstinence. ${ }^{12}$ In alcohol-dependent subjects, the perception of sleeping badly predicted relapse to heavy drinking at 12 -week follow-up. ${ }^{13}$

One interesting paradigm that has been suggested to instantiate mental fatigue is continued task repetition. Assuming subjects have no additional incentive to maintain consistently high performance, ${ }^{14}$ studies have shown that task performance and/or behavioral adjustment generally declines with increasing time spent performing a task ${ }^{15-18}$ findings interpreted as reflecting mental fatigue. Notably, this time-on-task performance erosion often occurs in conjunction with disengagement of the anterior cingulate cortex (ACC), as indicated by reductions in the scalp-recorded error-related negativity $^{15,16,18}$ (an event-related potential thought to be generated by the ACC, ${ }^{19}$ especially by its more cognitive dorsal (dACC) subregion, ${ }^{20}$ which spikes during error, negative feedback or high response conflict ${ }^{21}$ ). These findings could indicate that increasing time-on-task, and the mental fatigue that ostensibly ensues, weaken cognitive oversight functions of the dACC that encompass performance monitoring, ${ }^{22,23}$ cognitive control ${ }^{24}$ or signaling the need for increased attentional resources to enhance control. ${ }^{25}$ With increasing

\footnotetext{
${ }^{1}$ Department of Medical Research, Center for Translational Neuroimaging, Brookhaven National Laboratory, Upton, NY, USA; ${ }^{2}$ National Institute on Alcohol Abuse and Alcoholism, Bethesda, MD, USA; ${ }^{3}$ SUNY at Stony Brook, Stony Brook, NY, USA and ${ }^{4}$ National Institute on Drug Abuse, Bethesda, MD, USA

Correspondence: Dr RZ Goldstein, Department of Medical Research, Center for Translational Neuroimaging, Brookhaven National Laboratory, PO Box 5000,30 Bell Avenue, Upton, NY 11973-5000, USA.

E-mail: rgoldstein@bnl.gov

Keywords: anterior cingulate cortex; color-word stroop; dopamine; executive function; fMRI; methylphenidate; midbrain; motivation; positron emission tomography; self-regulation

Received 11 April 2012; revised 6 September 2012; accepted 6 September 2012
} 
disengagement of the $\mathrm{dACC}$, additional, compensatory regions may need to be recruited for task completion. Although time-on-task effects could stem from cognitive processes beyond fatigue, alternative processes including practice effects or target familiarity would ostensibly improve performance.

One such compensatory region, at least in healthy individuals, could be the dopaminergic midbrain, location of the ventral tegmental area and substantia nigra (which together are main sources of dopamine projection to the prefrontal cortex). This region is a major source for the release of dopamine, which has core roles in reward prediction, ${ }^{26,27}$ conditioning, ${ }^{28}$ incentive salience, ${ }^{29}$ arousal, ${ }^{30}$ novelty ${ }^{31,32}$ and aversion. ${ }^{33,34}$ In addition, dopamine supports numerous higher-order cognitive functions that bear on self-regulation and top-down control, encompassing cognitive flexibility, ${ }^{35}$ exertion and sustaining effort, ${ }^{36}$ and effort-based decisionmaking (for example, selecting rewards that are less desirable, but easier to obtain ${ }^{37-39}$ ). Of particular relevance to the current study, several lines of evidence have hinted that the dopaminergic midbrain may support endogenous motivation. For example, (A) a study of non-human primates performing an instrumental-conditioning task showed that midbrain dopamine firing was associated with animals' faster reaction time (RT), a marker of enhanced motivation; ${ }^{40}(\mathrm{~B})$ a different marker of task-related motivation (enhanced long-term memory for scenes) was associated with midbrain activity (to cues that signaled availability of high reward) in a human functional magnetic resonance imaging (fMRI) study; ${ }^{41}$ (C) a recent human $\mathrm{fMRI}$ study documented endogenous midbrain activity (that is, in the absence of extrinsic reward) during demanding trials on a visual discrimination task in healthy individuals; ${ }^{42}$ and (D) dopamine D2/D3 receptor availability in the midbrain (and nucleus accumbens), measured with positron emission tomography (PET), positively correlated with higher trait motivation (an achievement-oriented personality). ${ }^{43}$ Moreover, previous studies have hinted that dopaminergic mechanisms may underlie the mental fatigue-drug use relationship. For example, sleep deprivation enhanced the effect of cocaine on stereotyped behaviors in rats, interpreted to reflect hypersensitivity of dopaminergic receptors; ${ }^{44}$ similar dopaminergic hypersensitivity following sleep deprivation has been observed in healthy humans. ${ }^{45}$ Finally, under conditions of sleep deprivation, a critical node of the mesolimbic dopamine pathway (the nucleus accumbens) showed increased activity during risky decisions. ${ }^{46}$

The goals of the present study were to model mental fatigue as a function of time-on-task (repetition) in a cocaine-addicted population, and to probe for potential compensatory regions (and underlying neurotransmitters) (in both addicted individuals and healthy controls). Human volunteers performed an event-related color-word Stroop task, ${ }^{47}$ a classical inhibitory/ cognitive control task, widely used in fMRI environments. ${ }^{48-54}$ We hypothesized that time-on-task would produce more errors, less post-error slowing (that is, an increase in RT following an error, indicative of more controlled responding to prevent future errors ${ }^{55}$ ) and less dACC activity, together reflecting mental fatigue. In parallel, we expected that the midbrain would increase error-related activity as a function of time-on-task in healthy individuals, but not in cocaine abusers in whom dopamine neurotransmission is disrupted. ${ }^{56}$ Insofar as this midbrain response is marking enhanced task-related effort/motivation, ${ }^{40}$ we expected this region to correlate with faster RT. We did not expect a similar midbrain increase during successful resolution of cognitive conflict, as mental fatigue likely would be less prominently expressed during a successful trial.

Attribution of such midbrain activity to dopamine during this study was further tested (beyond what can be concluded through studying addicted individuals) through preliminary PET data and pharmacological manipulation. Data using PET with raclopride, a radioligand that competes with endogenous dopamine for binding to dopamine D2/D3 receptors and more directly measures dopaminergic neurotransmission in humans, were collected in a subset of our sample. We hypothesized that increased error-related midbrain fMRI activation would positively correlate with higher dopamine D2 receptor availability. In a second sample of subjects, pharmacological manipulation during $\mathrm{fMRI}$ was accomplished with an indirect dopamine agonist (oral methylphenidate). As dopamine exerts inverted U-shaped effects on cognition, ${ }^{57}$ benefiting those with initially decreased dopamine functioning (for example, impulsive individuals) but impairing those with initially optimal dopamine functioning (for example, nonimpulsive individuals), ${ }^{58}$ we hypothesized that methylphenidate would remediate this reduced midbrain response in cocaine abusers but would degrade this response in healthy controls. Effects of methylphenidate would both provide further evidence that effects are attributable to dopamine and suggest an avenue through which an aberrant midbrain response in addicted individuals could be normalized.

\section{Methods}

Subjects. Fifty-three healthy human volunteers (33 cocaine abusers and 20 healthy controls) who received no medication comprised our main sample (Sample 1). These 53 subjects had available fMRI scans from the first and last (fourth) repetitions of an event-related color-word Stroop task; these subject groups could also be matched demographically (Table 1) (see Supplementary Material for exclusion criteria and subject recruitment). A comprehensive diagnostic interview revealed that the cocaine subjects met criteria for current cocaine dependence $(N=22)$, cocaine dependence in partial remission $(N=3)$, cocaine dependence in fully sustained remission $(N=3)$, cocaine abuse $(N=4)$ or past polysubstance abuse with cocaine as the primary substance $(N=1)$ (see Supplementary Material for interview components and subject comorbidities).

In addition to Sample 1, we studied a secondary sample (Sample 2) that included 14 cocaine abusers and 15 healthy controls, each administered oral methylphenidate $(20 \mathrm{mg})$ or placebo on two separate, counterbalanced study days. Sample 2 subjects completed an fMRI color-word Stroop task with the same task parameters, except that this Stroop task included three repetitions (instead of four) to ensure that peak methylphenidate effects were sustained throughout (see Supplementary Material for complete methylphenidate procedures). These cocaine subjects met criteria for cocaine 


\begin{tabular}{|c|c|c|c|c|c|c|}
\hline & \multicolumn{3}{|c|}{ Sample 1} & \multicolumn{3}{|c|}{ Sample 2} \\
\hline & Test & $\begin{array}{l}\text { Cocaine } \\
\mathrm{N}=33\end{array}$ & $\begin{array}{l}\text { Control } \\
\mathrm{N}=20\end{array}$ & Test & $\begin{array}{l}\text { Cocaine } \\
\mathrm{N}=14\end{array}$ & $\begin{array}{l}\text { Control } \\
\mathrm{N}=15\end{array}$ \\
\hline Gender: male/female & $\chi^{2}=0.0$ & $28 / 5$ & $17 / 3$ & $\chi^{2}=1.1$ & $13 / 1$ & $15 / 0$ \\
\hline Race: African-American/Other & $\chi^{2}=2.5$ & $25 / 8$ & $11 / 9$ & $\chi^{2}=1.2$ & $12 / 2$ & $11 / 4$ \\
\hline Age (years) & $t=2.7^{*}$ & $44.2 \pm 6.3$ & $39.8 \pm 5.0$ & $t=2.7^{*}$ & $45.8 \pm 8.3$ & $38.6 \pm 6.1$ \\
\hline Education (years) & $t=1.9$ & $12.8 \pm 1.9$ & $13.8 \pm 1.7$ & $t=1.9$ & $12.9 \pm 2.2$ & $14.2 \pm 1.4$ \\
\hline $\begin{array}{l}\text { Verbal IQ: Wide Range Achievement Test III-Reading } \\
\text { Scale }^{92}\end{array}$ & $t=0.9$ & $94.0 \pm 11.9$ & $97.4 \pm 14.5$ & $t=1.6$ & $95.0 \pm 10.6$ & $101.2 \pm 9.7$ \\
\hline Non-verbal IQ: WASI-Matrix Reasoning Scale 93 & $t=0.8$ & $9.9 \pm 3.4$ & $10.6 \pm 2.8$ & $t=0.5$ & $10.6 \pm 2.8$ & $10.0 \pm 3.7$ \\
\hline Depression: Beck Depression Inventory II $^{94}$ & $Z=-1.6$ & $5.5 \pm 4.6$ & $3.6 \pm 3.6$ & $\mathrm{Z}=-3.1^{*}$ & $8.0 \pm 5.0$ & $2.2 \pm 4.1$ \\
\hline Socioeconomic status: Hollingshead Index & $t=1.0$ & $29.5 \pm 12.5$ & $32.7 \pm 12.9$ & $t=0.6$ & $36.3 \pm 9.8$ & $38.7 \pm 10.6$ \\
\hline Cigarette smokers (current or past/nonsmokers) & $\chi^{2}=14.2^{*}$ & $27 / 6$ & $6 / 14$ & $\chi^{2}=9.9^{*}$ & $11 / 3$ & $3 / 12$ \\
\hline $\begin{array}{l}\text { Daily cigarettes (current smokers: Sample 1: } N=23 / 4 \text {; } \\
\text { Sample } 2: N=10 / 0 \text { ) }\end{array}$ & $t=0.1$ & $8.7 \pm 5.8$ & $9.0 \pm 6.1$ & - & $8.4 \pm 4.1$ & - \\
\hline Time since last use (within $4 \mathrm{~h} />4 \mathrm{~h}$ ) & $\chi^{2}=4.6$ & $18 / 5$ & $1 / 3$ & - & $2 / 8$ & \\
\hline Age of onset (years) & - & $24.2 \pm 5.5$ & - & - & $26.6 \pm 8.2$ & - \\
\hline Duration of use (years) & - & $18.0 \pm 6.2$ & - & - & $14.5 \pm 8.1$ & - \\
\hline Current abstinence (days since last use) & - & $157.7 \pm 534.9$ & - & - & $3.9 \pm 6.7$ & - \\
\hline Cocaine urine status: positive/negative & - & $20 / 13$ & & & & \\
\hline Past month use: days/week & - & $2.6 \pm 2.4$ & - & - & $2.4 \pm 1.8$ & - \\
\hline Severity of Dependence Scale (0-15) & - & $6.2 \pm 4.0$ & - & - & $6.8 \pm 2.4$ & - \\
\hline Withdrawal symptoms: 18-item CSSA $(0-126)^{95}$ & - & $14.1 \pm 9.4$ & - & - & $17.0 \pm 7.2$ & - \\
\hline Cocaine craving: 5 -item Questionnaire $(0-45)^{96}$ & - & $16.4 \pm 12.2$ & - & - & $21.8 \pm 12.7$ & - \\
\hline
\end{tabular}

Abbreviations: CSSA, the Cocaine Selective Severity Assessment Scale; WASI, Wechsler Abbreviated Scale of Intelligence.

Note: values are frequencies or means \pm s.d.; ${ }^{*} P<0.05$; race: Other (Caucasian, Hispanic or Asian); $\chi^{2}$-tests were used for categorical variables; Mann-Whitney $U$ for all drug-related variables (continuous non-normally distributed variables); and $t$-tests for continuous, normally distributed variables.

dependence $(N=14)$ or cocaine abuse in partial remission $(N=1)$.

Task and behavioral measures. During the event-related fMRI Stroop task (Figure 1), ${ }^{48}$ subjects pressed for ink color of color words printed in either their congruent or incongruent colors. Four colors and words (red, blue, yellow and green) were used in all possible combinations. Both congruent and incongruent stimuli were presented randomly, except that no word or color of an incongruent stimulus was the same as the preceding congruent color word to avoid a priming effect. Each word was presented for $1300 \mathrm{~ms}$, with an intertrial interval of $350 \mathrm{~ms}$. Incongruent events were pseudorandomly spaced by at least five stimuli (range: 5-31; median: 14). Each run was $5.6 \mathrm{~min}$ (3300 ms for all stimuli, preceded by and terminating in fixation slides, 350 and $3200 \mathrm{~ms}$, respectively). Sample 1 subjects performed four consecutive runs of this task; Sample 2 subjects performed three runs. As there were 12 incongruent events in each run of 200 events, each subject's data contained up to 48 incongruent events. Accuracy, RT, post-conflict slowing and post-error slowing were collected using E-prime (see Supplementary Material on how the latter two variables were calculated). To bolster the case for fatigue, we also inspected self-reported ratings of sleepiness and task interest (collected as part of a different, preceding drug Stroop task; see Supplementary Material for ratings procedures).

MRI data acquisition. MRI scanning was performed on a whole-body MRI scanner composed by a Varian INOVA console (Palo Alto, CA, USA) interconnected to a Siemens 4Tesla Interatom magnet (Erlangen, Germany). The fMRI blood-oxygenation-level-dependent (BOLD) responses were measured as a function of time using a $\mathrm{T}^{*}$-weighted singleshot gradient-echo EPI sequence (TE/TR $=20 / 1600 \mathrm{~ms}, 4 \mathrm{~mm}$ slice thickness, $1 \mathrm{~mm}$ gap, typically 33 coronal slices, $20 \mathrm{~cm}$ FOV, $64 \times 64$ matrix size, $90^{\circ}$-flip angle, $200 \mathrm{kHz}$ bandwidth with ramp sampling, 207 time points and 4 dummy scans to avoid non-equilibrium effects in the fMRI signal). Padding minimized subject motion, which was also monitored immediately after each $\mathrm{fMRI}$ run. ${ }^{59}$ Earplugs ( $28 \mathrm{~dB}$ sound attenuation; Aearo Ear TaperFit 2, 3M Co., St Paul, MN, USA) and headphones (30 dB sound attenuation; Commander XG MRI Audio System, Resonance Technology Inc., Northridge, CA, USA) minimized scanner noise. ${ }^{60}$ Anatomical images were collected using a T1-weighted 3D-MDEFT (three-dimensional modified driven equilibrium Fourier transform) sequence ${ }^{61}$ and a modified T2-weighted hyperecho sequence, ${ }^{62}$ inspected by a neurologist for gross morphological abnormalities.

MRI data processing. Image reconstruction was performed using an iterative phase correction method that produces minimal signal-loss artifacts in echo-planar images. ${ }^{63}$ Subsequent analyses were performed with statistical parametric mapping (SPM2) (Wellcome Trust Center for Neuroimaging, London). A six-parameter rigid body transformation (three rotations, three translations) was used for image realignment and for correction of head motion. Criteria for acceptable motion were displacement $<2 \mathrm{~mm}$ and rotation $<2 \circ$. The realigned data sets were spatially normalized to the standard stereotactic space of the Montreal Neurological Institute (MNI) using a 12-parameter affine transformation ${ }^{64}$ and a voxel size of $3 \times 3 \times 3 \mathrm{~mm}^{3}$. An $8-\mathrm{mm}$ full-width-halfmaximum Gaussian kernel was for spatial smoothing. 




b

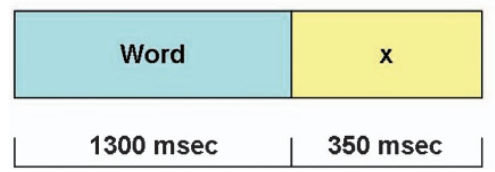

C



Figure 1 Functional magnetic resonance imaging (fMRI) color-word Stroop task. Subjects pressed for ink color as quickly and accurately as possible (performance was recorded throughout). fMRI response to conflict trials (all incongruent), error trials (all error) and their interaction were each compared with active baselines (all congruent trials, all correct trials and congruent correct trials, respectively). (a) Examples of color words: the circled (red) stimulus is congruent; all others are incongruent. (b) Individual trial, comprised of a 1300-ms color-word stimulus and $350 \mathrm{~ms}$ interstimulus interval. (c) Individual run, comprised of 200 individual trials and a $3200-\mathrm{ms}$ interval to separate runs.

BOLD-fMRI analyses. Three general linear models, ${ }^{65}$ each with six motion regressors (three translation and three rotation) and up to three task conditions (incongruent correct events, congruent error events and/or incongruent error events) convolved with a canonical hemodynamic response function and low-pass and high-pass (cutoff frequency: 1/ $90 \mathrm{~s}$ ) filters, were used to calculate individual BOLD-fMRI maps. Contrast maps were calculated for all available runs for all subjects (who met motion criteria as described above), with each contrast reflecting percent signal change from baseline. The baselines of these three models consisted of all the task events that were not modeled in the relevant design matrices, and at minimum included the fourth (and most frequent) type of task event (congruent correct events). Specifically, Design Matrix 1 included one regressor collapsed across both incongruent trials (Incongruent Correct and Incongruent Incorrect), leaving out both congruent trials (Congruent Correct and Congruent Incorrect) to serve as the baseline. Design Matrix 1 tested for a main effect of 'congruency', defined as (Incongruent Error + Incongruent Correct) - (Congruent Error + Congruent Correct). Design Matrix 2 included one regressor collapsed across both error trials (Congruent Incorrect and Incongruent Incorrect), leaving out both correct trials (Congruent Correct and Incongruent Correct) to serve as the baseline. Design Matrix 2 tested for a main effect of 'correctness', defined as (Incongruent Error + Congruent Error) - (Incongruent Correct + Congruent Correct). Design Matrix 3 included three regressors: Incongruent Correct trials, Congruent Incorrect trials and Incongruent Incorrect trials, leaving out the Congruent Correct trials to serve as the baseline. Design Matrix 3 tested for a 'correctness $\times$ congruency' interaction, defined as ((Incongruent Correct - Congruent Correct) - (Incongruent Error Congruent Correct $))+($ Congruent Error-Congruent Correct). In these 1st level analyses, each of these three contrasts was computed separately for each task repetition.

At the 2nd level, we tested how each of these 1st level contrasts differed as a function of repetition and group. To maximize sample size, Sample 1 analyses principally compared the first and fourth task runs (repetitions). We estimated three separate 2 (repetition: first, last) $\times 2$ (group: cocaine, control) mixed analyses of variance at the wholebrain level in SPM2; however, to provide support for graded effects, we also inspected all four repetitions among subjects with complete fMRI data (Supplementary Material). Sample 2 analyses principally contrasted the first and third task runs; however, the second task repetition was also examined (Supplementary Material). Sample 2 analyses also included an additional 2nd level within-subjects factor: medication condition (methylphenidate or placebo). To maximize the number of available Sample 2 subjects, we analyzed all subjects who had usable fMRI scans from at least $3 / 4$ task conditions (first and third task repetitions, for methylphenidate and placebo study days). These 29 subjects were missing 12/ 116 scans (10.3\%); 17 of these subjects (10 cocaine subjects and 7 healthy controls) had complete data (all four scans). As the neuroimaging results were unchanged when analyzing either the 29 subjects with some missing data or the 17 subjects with complete data, we elected to report results from the larger sample.

fMRI activations from peak coordinates were further extracted using the SPM EasyMRI toolbox and verified for anatomical accuracy using MRIcron. These extracted BOLDfMRI signals were used to inspect for outliers and to conduct correlation analyses in SPSS. These correlation analyses tested for respective associations between select activations (that is, regions that showed significant main effects or interactions of repetition as reported in Results) and select behavioral measures (that is, errors, RT and post-error slowing), separately during the first and last task runs. For Sample 2, correlation analyses were also split by medication. Task performance behavioral analyses were considered significant at $P<0.05$. SPM2 analyses were considered significant at $P_{\text {corr }}<0.05$ (cluster-level $P<0.05$ family-wise error corrected threshold, search threshold of $P<0.005$ voxel uncorrected, 15 contiguous voxels). As a large number of brain-behavior associations were available for inspection, correlation analyses were considered significant at of $P<0.01$ (a more conservative threshold to minimize Type I errors). Given the complexity of the current design and approach, such brain-behavior correlations are essential to increase understanding of the underlying mechanisms.

Preliminary PET data. A subsample of Sample 1, consisting of five cocaine subjects and seven controls (who did not differ from the larger sample on any demographic, drug use 
or task performance variables (all $P>0.05$, independent samples $t$-tests)), was also scanned with PET (raclopride imaging for dopamine D2 receptor availability in the striatum). This sample size, although not large, is comparable to some of the previous studies assessing correlations between fMRI BOLD and PET (for example, $N=11 ;{ }^{66} N=18^{67}$ ).

PET imaging was performed on a Siemens $\mathrm{HR}+$ tomograph (resolution: $4.5 \times 4.5 \times 4.5 \mathrm{~mm}^{3}$ full-width half-maximum). Dynamic scans were started immediately after injection of $4-10 \mathrm{mCi}\left[{ }^{11} \mathrm{C}\right]$ raclopride (specific activity $0.5-$ $1.5 \mathrm{Ci} / \mu \mathrm{m}$ at end of bombardment) and were obtained for 60 min. $^{68}$ Arterial blood was obtained to measure the concentration of unchanged $\left[{ }^{11} \mathrm{C}\right]$ raclopride in plasma. Regions of interest were obtained in striatum (caudate, putamen and ventral striatum) and cerebellum (reference region). ${ }^{43}$ The distribution volumes in these regions were computed as the ratio of tissue radiotracer concentration to plasma non-metabolized radiotracer concentration. The ratio of distribution volume in the striatum to that of the cerebellum corresponds to $\left(B_{\max } / K d\right)+1$ and was used as measures of D2/D3 receptor availability (non-displaceable binding potential). We predicted that midbrain (but not dACC) fMRI activity would positively correlate with striatal dopamine $\mathrm{D} 2$ receptor availability (note that consistent with previous PET studies, ${ }^{56}$ this measure was higher in controls $(2.5 \pm 0.1)$ than in cocaine subjects $(2.1 \pm 0.1), t(10)=-2.2, P=0.05))$. As such dopaminergic neurotransmission correlates with an achievementoriented personality, ${ }^{43}$ current PET data may be viewed as signifying trait motivation (complementing findings of our behavioral marker of motivation, RT).

\section{Results}

Sample 1: behavior. Behavioral data (errors and RT) were (separately) analyzed with 2 (congruency: congruent, incongruent) $\times 2$ (repetition: first, fourth) $\times 2$ (group: cocaine, control) analyses of variances. As predicted, all subjects committed more errors during the fourth than the first repetition (repetition main effect: $F(1,51)=21.0, P<0.001$ ). A repetition $\times$ congruency interaction $(F(1,51)=23.8$, $P<0.001$ ), followed by post-hoc tests, revealed that all subjects committed more errors during the fourth than first repetition during the congruent trials $(t(52)=5.1, P<0.001)$ (Figure 2a) but not during the incongruent trials $(t(52)=0.4$, $P>0.7)$. Results of RT revealed the classical Stroop interference effect (faster RT on congruent trials than incongruent trials: $F(1,51)=332.0, P<0.001)$. No other main effects or interactions emerged for errors or RT.

Post-conflict slowing and post-error slowing were separately analyzed with 2 (repetition) $\times 2$ (group) analyses of variances (post-error slowing was analyzed separately for congruent trials, incongruent trials and their sum; see Supplementary Material for further discussion). Post-error slowing (when the trial after the error was a congruent trial) was higher during the first than the fourth task repetition $(F(1,45)=11.4, \quad P<0.01) \quad$ (Figure 2b). Although the repetition $\times$ group interaction was initially significant $(F(1,45)=6.0, \quad P<0.05)$, such that post-error slowing decreased as a function of time-on-task only in controls (paired $t(18)=5.6, P<0.001$ ) (an effect likely attributable to baseline group differences, insofar as post-error slowing was lower in cocaine subjects than controls during the first repetition; independent $t(47)=2.3, P<0.05)$, this interaction did not survive correction for covariates (Supplementary Material) and is not interpreted. There were no effects for post-error slowing during incongruent trials, possibly because of their infrequency or for post-conflict slowing (that is, increased RT following an incongruent, but correct, trial). Supplementary Table S1 (Supplementary Material) presents the Sample 1 means and s.e. of all behavioral variables.
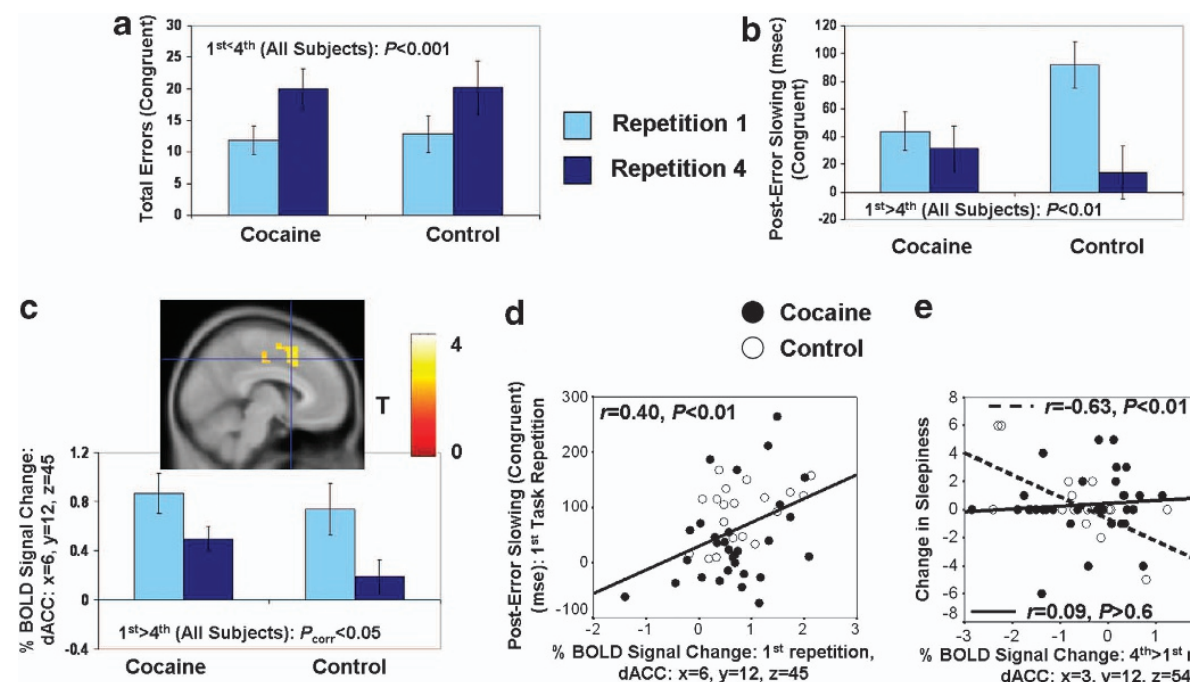

e

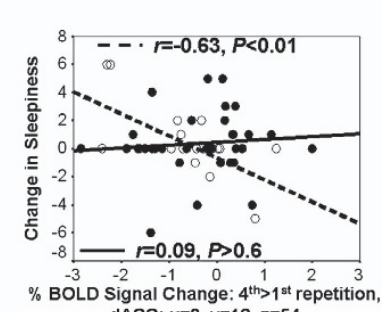

Figure 2 Evidence of mental fatigue as a function of time-on-task. During the event-related color-word Stroop task, performance on the fourth repetition (compared with the first) produced (a) more congruent errors, (b) less post-error slowing on congruent trials and (c) less \% blood-oxygenation-level-dependent (BOLD) signal change to error (compared with all correct trials) in the dorsal anterior cingulate cortex (dACC). (d) Scatterplot showing that higher error-induced BOLD signal change during the first task repetition correlated with respective longer post-error slowing in all subjects. (e) Scatterplot showing that the higher the change in dACC activity (fourth $>$ first repetition), the higher was the sleepiness before beginning the task. Asterisks denote a significant difference between first and last task runs (repetitions). 


\section{Sample 1: SPM}

Conflict. During conflict, whole-brain SPM analyses revealed repetition main effects in the thalamus (first $>$ fourth), precentral gyrus (first <fourth) and parietal cortex (first $<$ fourth); cocaine subjects also showed more activity than controls in lingual gyrus and cerebellum (Table 2). However, because there were no repetition $\times$ group interactions for the 'congruency' main effect contrast, subsequent analyses focused on 'correctness' activations as described below. Importantly, there were also no activations for the 'correctness $\times$ congruency' interaction, indicating that effects during error were not further modulated by conflict.

Error. During error, whole-brain SPM analyses revealed decreased activity with repetition in all subjects in the right dACC (Figure 2c), a cluster that extended dorsally to the bilateral supplementary motor area (SMA) (Table 2). The higher the extracted BOLD signals in the right dACC (but not SMA) (that is, the peak dACC coordinate from Table 2) during the first task repetition, the higher was the respective post-error slowing in all subjects (Figure 2d; note that this correlation remained significant after excluding the potential outlier in the bottom left quadrant). Thus, both behavior and brain measures on the task were consistent with mental fatigue (and not alternative processes such as practice effects), and were interrelated across all subjects. Further supporting this interpretation of mental fatigue, the higher the increase in self-reported sleepiness (but not task interest) during a preceding drug Stroop task, the higher was the decrease (fourth > first repetition) in dACC/SMA activity during the color-word Stroop task in controls (though not in cocaine subjects) (Figure 2e). Thus, healthy individuals who showed the greatest decrease in dACC/SMA activity during the color-word Stroop task were also the ones likely fatigued before beginning the task.

Importantly, whole-brain SPM analyses also revealed a repetition $\times$ group interaction in the right midbrain during error (that extended dorsally to include part of the thalamus; Table 2): cocaine subjects showed decreased error-induced BOLD signal in this region with repetition, whereas the controls showed the opposite pattern (Figure 3a). Peak activity of this cluster did not appear to include the locus coeruleus (Supplementary Material). During the fourth repetition, the BOLD signal extracted from the midbrain (the peak coordinate from Table 2) correlated with faster RT in all subjects (Figure $3 b$ ) and higher striatal dopamine D2 receptor availability in caudate in all subjects (after excluding one outlier) (Figure 3c). This PET dopamine measure did not correlate with fMRI dACC or SMA activity $(P>0.4)$. Thus, fMRI midbrain activity (but not dACC activity) during the last task repetition correlated with both state and trait measures of motivation in all subjects.

Sample 2: SPM. For these Sample 2 subjects, we restricted repetition-associated analyses to midbrain activity during error because of our targeted a priori hypotheses and the

Table 2 Color-word Stroop SPM error- and conflict-related activations (vs all correct activations) during the first and last task repetitions

\begin{tabular}{|c|c|c|c|c|c|c|c|c|}
\hline & $B A$ & Side & Voxels & Peak Z & $\begin{array}{l}P \text { cluster-level } \\
\text { corrected }\end{array}$ & $x$ & $y$ & $z$ \\
\hline \multicolumn{9}{|c|}{ SAMPLE 1} \\
\hline dACC/supplementary motor area & 6,32 & B & 178 & 3.8 & 0.029 & $\begin{array}{r}3 \\
6 \\
-12\end{array}$ & $\begin{array}{l}12 \\
12 \\
15\end{array}$ & $\begin{array}{l}54 \\
45 \\
51\end{array}$ \\
\hline \multicolumn{9}{|c|}{ Error: repetition (first, fourth) $\times$ group (cocaine, control) interaction } \\
\hline $\begin{array}{l}\text { Midbrain: VTA/STN complex } \\
\text { Thalamus }\end{array}$ & - & $\mathrm{R}$ & 183 & 4.5 & 0.026 & $\begin{array}{r}6 \\
12\end{array}$ & $\begin{array}{l}-18 \\
-15\end{array}$ & $\begin{array}{r}-9 \\
0\end{array}$ \\
\hline \multicolumn{9}{|c|}{ Conflict: repetition main effect (first $>$ fourth) } \\
\hline Thalamus & - & B & 372 & 4.2 & 0.001 & $\begin{array}{r}15 \\
-6\end{array}$ & $\begin{array}{l}-27 \\
-15\end{array}$ & $\begin{array}{l}0 \\
9\end{array}$ \\
\hline \multicolumn{9}{|c|}{ Conflict: repetition main effect (first $<$ fourth) } \\
\hline Precentral gyrus & 6,4 & $\mathrm{R}$ & 162 & 3.6 & 0.042 & $\begin{array}{l}33 \\
36\end{array}$ & $\begin{array}{l}-12 \\
-24\end{array}$ & $\begin{array}{l}48 \\
60\end{array}$ \\
\hline $\begin{array}{l}\text { Inferior parietal lobule } \\
\text { Superior parietal lobule }\end{array}$ & 40,7 & $\mathrm{~L}$ & 288 & 3.5 & 0.003 & $\begin{array}{l}-39 \\
-27\end{array}$ & $\begin{array}{l}-48 \\
-60\end{array}$ & $\begin{array}{l}51 \\
57\end{array}$ \\
\hline \multicolumn{9}{|c|}{ Conflict: group main effect (cocaine > control) } \\
\hline $\begin{array}{l}\text { Lingual gyrus } \\
\text { Cerebellum }\end{array}$ & 18,37 & L & 203 & 3.7 & 0.016 & $\begin{array}{l}-12 \\
-21\end{array}$ & $\begin{array}{l}-66 \\
-57\end{array}$ & $\begin{array}{r}-9 \\
-15\end{array}$ \\
\hline \\
\hline Midbrain: VTA/STN complex ${ }^{\mathrm{a}}$ & - & $\mathrm{B}$ & 38 & 4.5 & 0.002 & -3 & -18 & -15 \\
\hline
\end{tabular}

Abbreviations: B, bilateral (neurological convention); BA, Brodmann Area; dACC, dorsal anterior cingulate cortex; L, left side, R, right side; STN, substantia nigra; VTA, ventral tegmental area.

Note: regions significant at $P_{\text {corr }}<0.05$ (cluster-level corrected for multiple comparisons, 15 voxels minimum).

${ }^{a}$ Used a bilateral $10 \mathrm{~mm}$ spherical mask around the peak midbrain coordinate of Sample 1 ( $x=|6|, y=-18, z=-9$ ); the Stroop task completed by Sample 1 had four repetitions, while the Stroop task completed by Sample 2 had three repetitions. 
a



d

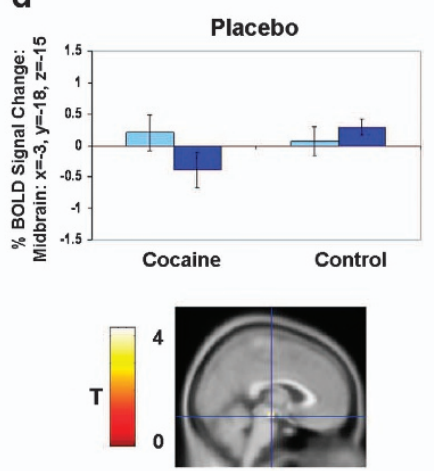

Control
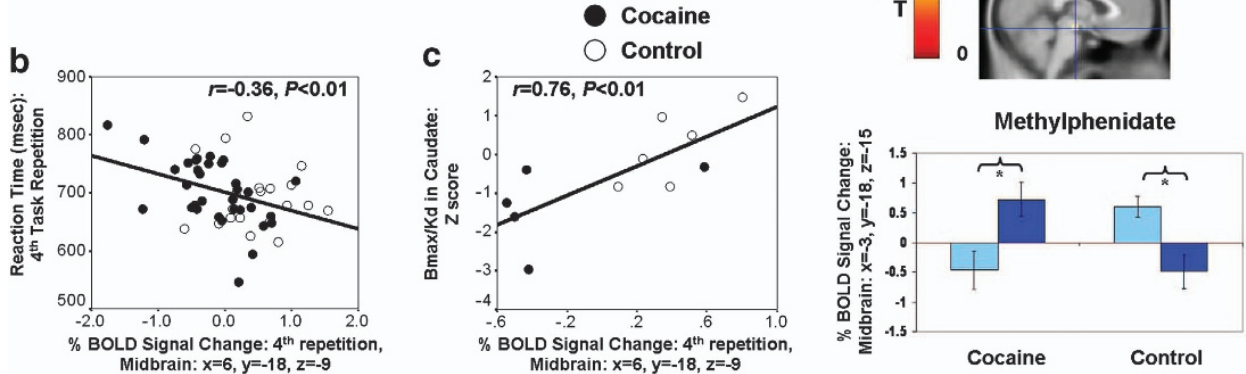

Figure 3 Midbrain response to error as a function of time-on-task. (a) More \% blood-oxygenation-level-dependent (BOLD) signal change to error (compared with all correct trials) in the midbrain during the fourth task repetition in controls, but during the first task repetition in the cocaine subjects. Such higher error-induced BOLD signal change during the fourth task repetition correlated with (b) faster reaction time (RT) and (c) dopamine D2 receptor availability in caudate, as measured by positron emission tomography (PET) (one outlier was excluded; for display purposes, the scores of the cocaine subjects were standardized to the mean and s.d. of controls). (d) Midbrain responses as a function of time-on-task were reversed in both study groups during methylphenidate (note that the correct congruent baseline means that BOLD responses below zero do not necessarily indicate deactivations). Asterisks denote a significant difference between first and last task runs (repetitions).

smaller sample size. We also used a bilateral spherical mask of the midbrain coordinates from Sample 1, with a search threshold set at the more conservative $P_{\text {FWE }}<0.05$ (voxellevel family-wise error correction) (cluster extent remained at 15 voxels). Consistent with hypotheses, SPM analyses revealed a repetition (first, third) $\times$ medication (methylphenidate, placebo) $\times$ group (control, cocaine) three-way interaction in the bilateral midbrain (Table 2): during placebo, the pattern of results mirrored Sample 1; during methylphenidate, however, the pattern of effects was reversed, such that the cocaine subjects increased error-related activity in the midbrain with repetition, whereas the controls decreased activity in this context (Figure 3d). Again, these effects did not extend to the locus coeruleus (Supplementary Material). We did not replicate the midbrain correlation with RT during the last task repetition (here, the third repetition), during either methylphenidate or placebo. For behavioral task performance, there were no modulatory effects of methylphenidate on task repetition that survived correction for covariates (Supplementary Material).

\section{Discussion}

Cocaine abusers and healthy controls completed a laboratory paradigm that aimed to model mental fatigue as a function of time-on-task. Support for such fatigue in Sample 1 came from measures of behavior (more errors, less post-error slowing) and brain function (less dACC activity to error, which could indicate decreased cognitive control ${ }^{24}$ and/or performance monitoring ${ }^{22,23}$ ); the latter two variables were themselves positively correlated during the first repetition in all subjects.
At first blush, it might seem surprising that such results only emerged for the congruent trials. However, it is not entirely unexpected that mental fatigue would be best manifested during the easier and more frequent congruent trials. In further support, the higher the fatigue before beginning the task, the greater was the decrease in dACC activity throughout the task. These collective fMRI findings extend prior studies on repetition-induced fatigue in healthy controls that have traditionally relied on electroencephalogram methods. ${ }^{15-18}$ More generally, our findings support a suggested function of the ACC in shaping future behavior as a function of reward history, ${ }^{69}$ such that mental fatigue may ensue when the costs (that is, exertion) associated with continued task performance are calculated to outweigh the benefits (that is, reward). ${ }^{70}$ Interestingly, although prior work led us to anticipate reduced dACC error-related activity, and possibly more prominent fatigue, in the cocaine subjects, ${ }^{71}$ the $\mathrm{dACC}$ response to error decreased with task repetition to a similar extent in all subjects. One explanation for these null dACC activation differences between the groups could be the lack of respective differences in task performance. Consistent with this idea, previous studies have indicated that when errorrelated performance differs between the groups, dACC errorrelated activity differs in parallel. ${ }^{72-74}$ In contrast, and similarly to the current study, one study showed that when opiate addicted individuals and healthy controls did not exhibit performance differences on the Multi-Source Interference Task, group differences in the dACC were also nonsignificant. $^{75}$

The interesting dissociation between groups occurred in the dopaminergic midbrain. Healthy individuals increased 
midbrain activity to error when fatigue was putatively escalating, whereas cocaine abusers decreased midbrain activity to error in this context (possibly indicating an aberrant response in this latter group). These results extend the hypothesized function of dopamine in sustaining effort during task performance ${ }^{36-38}$ into human executive function, cognitive control and mental fatigue. These results help shed light on previous research involving midbrain morphological dysfunction in disease states characterized by fatigue. For example, decreased midbrain white matter volume was associated with a longer duration of illness in chronic fatigue syndrome, ${ }^{76}$ and polio survivors who later developed fatigue as a consequence of infection were found post-mortem to have lesions of various subcortical regions including the midbrain. ${ }^{77}$ Given the midbrain-RT correlation during the last task repetition, we interpret the midbrain activity in the current study as potentially marking sustained motivation-to our knowledge demonstrated for the first time during mental fatigue-that was presumably deployed when self-regulatory resources were needed most. This interpretation supports other neuroimaging studies of healthy individuals, which, although using different paradigms, have similarly implicated the midbrain in endogenous motivation. ${ }^{41-43}$ This interpretation also agrees with a body of exercise literature indicating that reduced dopamine neurotransmission may contribute to early-onset fatigue-possibly because of diminished motivation (reviewed in Davis and Bailey ${ }^{78}$ ); in contrast, increased exercise appears to facilitate midbrain dopamine neurotransmission as indicated by enhanced tyrosine hydroxylase mRNA expression and reduced D2 autoreceptor mRNA. ${ }^{79}$ Finally, our interpretation is consistent with recent theorizing on dopamine, where a subpopulation of dopamine neurons is hypothesized to respond to motivational salience (detecting and responding to important events, and providing impetus to persist toward desirable outcomes). ${ }^{80}$ Seemingly contradictory midbrain results in related studies (for example, deactivation of the midbrain after errors was observed using the stop signal paradigm, ${ }^{81}$ while midbrain activation was not reported in other studies that inspected Stroop repetition effects $^{52,82}$ ) are likely attributable to differences in task design (for example, shorter time course of interest: response after only $100 \mathrm{~ms}$ in the previous stop signal study) and dependent measures (for example, previous Stroop repetition studies investigated conflict, whereas we focused on error).

Our unique multimodal methodological approach, which included data from a relevant disease state (cocaine abusers), preliminary PET data and administration of a dopamine agonist, methylphenidate, collectively suggested that dopamine could underlie these midbrain effects. Indeed, cocaine subjects showed decreased dopamine D2 receptor availability compared with controls even in this small sample, increasing validity of using this population. Moreover, preliminary PET data, where higher midbrain signal to error during the last task repetition correlated with higher dopamine D2 receptor availability in caudate, directly linked the current midbrain error-related activity to dopamine neurotransmission. Finally, methylphenidate produced a striking reversal of midbrain effects in the cocaine subjects versus the controls. This latter finding supports dopamine's inverted U-shaped effects on cognition ${ }^{57}$ and brain activity, ${ }^{83}$ where increased extracellular dopamine, afforded here through methylphenidate, is hypothesized to selectively benefit those with the greatest need (possibly normalizing response in cocaine subjects due to restoration of dopamine signaling but degrading response in healthy controls due to excessive dopamine signaling). In agreement with this idea, dopamine agonists (for example, the dopamine D1/D2 receptor agonist pergolide) have been shown to improve fatigue in medically ill patients (for example, Parkinson's disease ${ }^{84}$ ), and stimulant medications (indirect dopamine agonists) have helped sustain cognitive and behavioral performance in healthy military personnel under conditions of extreme fatigue. ${ }^{85}$ Thus, our current findings highlight methylphenidate's potentially corrective impact on brain and behavior in cocaine addiction. ${ }^{86,87}$ In particular, methylphenidate may have helped normalize the dopaminergic perturbations that characterize cocaine addiction (for example, reduced dopamine receptor availability and release). ${ }^{56}$ Future clinical intervention studies will need to evaluate the therapeutic implications of these findings (for example, the possibility of enhancing motivation in addicted individuals during mental fatigue).

This study has several limitations. First, although previous research guided interpretations of the current activations, the correlational nature of fMRI constrains our ability to definitely conclude that a particular cognitive process (for example, mental fatigue) was engaged in this study. Other potential dACC-related cognitive processes may have included vigilance, attention or executive function decline. However, current brain-behavior correlations lessen this concern (for example, correlations between dACC and post-error slowing or sleepiness but not task interest, and between midbrain and RT). Moreover, the pattern of effects observed during task performance (increased errors with increased time-on-task during congruent trials) appears consistent with mental fatigue. Second, current PET results require replication with larger samples. Third, although we speculated that the easier and more frequent congruent trials were best suited for tapping into time-on-task effects as reflective of mental fatigue in the current task design, novelty may have also contributed to the null results during the (less frequent) incongruent trials, counteracting potentially similar mental fatigue effects during these more difficult trials. This consideration needs to be addressed with future tasks designed for this purpose. Fourth, although post-error slowing is thought to rely on top-down control processes, in the current study (after correction for covariates) post-error slowing did not differ between the groups as a function of time-on-task (Results) and did not correlate with task performance at the nominal $P<0.01$ statistical threshold for correlations (Supplementary Material), and therefore conclusions about this variable visà-vis cognitive functioning should be appropriately tempered. Nevertheless, given that the repetition $\times$ group interaction was initially significant (before correction for covariates), we would predict that larger sample sizes might be able to detect impairments in initial post-error slowing and other indicators of more careful task performance in addicted individuals. Fifth, because methylphenidate also blocks the norepinephrine transporter ${ }^{88}$ and because norepinephrine may contribute to motivated, reward-directed behaviors by modifying dopamine activity in the midbrain, ${ }^{89}$ the potential contribution of 
norepinephrine to these results awaits further study (for example, through administration of selective dopamine or norepinephrine agonists or antagonists). However, reducing this concern are: (A) PET data used raclopride, a selective dopamine antagonist; $(B)$ an region of interest analysis of the locus coeruleus did not reveal effects in either sample (Supplementary Material); (C) as discussed above, selective dopamine agonists have previously produced salubrious effects on fatigue; ${ }^{84}$ and (D) even if effects were due to norepinephrine transporter blockade, dopamine could still be involved since norepinephrine transporters also have affinity for dopamine. ${ }^{88}$

In summary, our results suggest the important and novel conclusion that a putatively healthy-and motivationally relevant-dopaminergic response to error during mental fatigue is compromised in individuals addicted to cocaine. Strengthening this response through pharmacological intervention, perhaps especially if combined with select cognitive exercises $^{90}$ or self-control interventions, ${ }^{91}$ might help addicted individuals sustain motivation both to engage in therapeutic activities and to maintain abstinence in situations that could trigger relapse.

\section{Conflict of interest}

The authors declare no conflict of interest.

Acknowledgements. This study was supported by grants from the National Institute on Drug Abuse (to RZG: 1R01DA023579; to SJM: 1F32DA030017-01) and the NIH Intramural program. We gratefully acknowledge the contributions of Muhammad A Parvaz, Anna Konova, Nelly Alia-Klein, Thomas Maloney, Patricia A Woicik, Ruiliang Wang, Alex Panagopoulos, Dimitris Samaras, Frank Telang and Gene-Jack Wang. This manuscript has been authored by Brookhaven Science Associates, LLC under Contract No. DE-AC02-98CHI-886 with the US Department of Energy. The United States Government retains, and the publisher, by accepting the article for publication, acknowledges, a world-wide license to publish or reproduce the published form of this manuscript, or allow others to do so, for the United States Government purposes.

1. Goldstein RZ, Volkow ND. Dysfunction of the prefrontal cortex in addiction: neuroimaging findings and clinical implications. Nat Rev Neurosci 2011; 12: 652-669.

2. Paulus MP. Decision-making dysfunctions in psychiatry-altered homeostatic processing? Science 2007; 318: 602-606.

3. Kalivas PW, Volkow ND. The neural basis of addiction: a pathology of motivation and choice. Am J Psychiatry 2005; 162: 1403-1413.

4. Salo R, Ursu S, Buonocore MH, Leamon MH, Carter C. Impaired prefrontal cortical function and disrupted adaptive cognitive control in methamphetamine abusers: a functional magnetic resonance imaging study. Biol Psychiatry 2009; 65: 706-709.

5. Heatherton TF, Wagner DD. Cognitive neuroscience of self-regulation failure. Trends Cogn Sci 2011; 15: 132-139.

6. Muraven M, Collins RL, Shiffman S, Paty JA. Daily fluctuations in self-control demands and alcohol intake. Psychol Addict Behav 2005; 19: 140-147.

7. Muraven M, Shmueli D. The self-control costs of fighting the temptation to drink. Psychol Addict Behav 2006; 20: 154-160.

8. Morgan PT, Malison RT. Cocaine and sleep: early abstinence. Sci World J 2007; 7: 223-230.

9. Aharonovich E, Nunes E, Hasin D. Cognitive impairment, retention and abstinence among cocaine abusers in cognitive-behavioral treatment. Drug Alcohol Depend 2003; 71: 207-211.

10. Aharonovich E, Hasin DS, Brooks AC, Liu X, Bisaga A, Nunes EV. Cognitive deficits predict low treatment retention in cocaine dependent patients. Drug Alcohol Depend 2006; 81: 313-322.

11. Hamidovic A, de Wit $H$. Sleep deprivation increases cigarette smoking. Pharmaco Biochem Behav 2009; 93: 263-269.
12. Okun ML, Levine MD, Houck P, Perkins KA, Marcus MD. Subjective sleep disturbance during a smoking cessation program: associations with relapse. Addict Behav 2011; 36: 861-864.

13. Foster JH, Peters TJ. Impaired sleep in alcohol misusers and dependent alcoholics and the impact upon outcome. Alcohol Clin Exp Res 1999; 23: 1044-1051.

14. Bonnefond A, Doignon-Camus N, Hoeft A, Dufour A. Impact of motivation on cognitive control in the context of vigilance lowering: an ERP study. Brain Cogn 2011; 77: 464-471.

15. Lorist MM, Boksem MA, Ridderinkhof KR. Impaired cognitive control and reduced cingulate activity during mental fatigue. Brain Res Cogn Brain Res 2005; 24: 199-205.

16. Boksem MA, Meijman TF, Lorist MM. Mental fatigue, motivation and action monitoring. Biol Psychol 2006; 72: 123-132.

17. Lorist MM, Bezdan E, ten Caat M, Span MM, Roerdink JB, Maurits NM. The influence of mental fatigue and motivation on neural network dynamics; an EEG coherence study. Brain Res 2009; 1270: 95-106.

18. Kato $\mathrm{Y}$, Endo $\mathrm{H}$, Kizuka T. Mental fatigue and impaired response processes: event-related brain potentials in a Go/NoGo task. Int J Psychophysiol 2009; 72: 204-211.

19. Dehaene S, Posner MI, Tucker DM. Localization of a neural system for error-detection and compensation. Psychol Sci 1994; 5: 303-305.

20. Amodio DM, Jost JT, Master SL, Yee CM. Neurocognitive correlates of liberalism and conservatism. Nat Neurosci 2007; 10: 1246-1247.

21. Jocham G, Ullsperger M. Neuropharmacology of performance monitoring. Neurosci Biobehav Rev 2009; 33: 48-60.

22. van Veen V, Carter CS. The anterior cingulate as a conflict monitor: $f M R I$ and ERP studies. Physiol Behav 2002; 77: 477-482.

23. MacDonald AW 3rd, Cohen JD, Stenger VA, Carter CS. Dissociating the role of the dorsolateral prefrontal and anterior cingulate cortex in cognitive control. Science 2000; 288: 1835-1838.

24. Ridderinkhof KR, Ullsperger M, Crone EA, Nieuwenhuis $\mathrm{S}$. The role of the medial frontal cortex in cognitive control. Science 2004; 306: 443-447.

25. Kerns JG, Cohen JD, MacDonald AW 3rd, Cho RY, Stenger VA, Carter CS. Anterio cingulate conflict monitoring and adjustments in control. Science 2004; 303: 1023-1026.

26. Montague PR, Berns GS. Neural economics and the biological substrates of valuation. Neuron 2002; 36: 265-284.

27. Schultz W. Dopamine signals for reward value and risk: basic and recent data. Behav Brain Funct 2010; 6: 24.

28. Hazy TE, Frank MJ, O'Reilly RC. Neural mechanisms of acquired phasic dopamine responses in learning. Neurosci Biobehav Rev 2010; 34: 701-720.

29. Berridge KC. The debate over dopamine's role in reward: The case for incentive salience. Psychopharmacology 2007; 191: 391-431.

30. Horvitz JC. Mesolimbocortical and nigrostriatal dopamine responses to salient non-reward events. Neuroscience 2000; 96: 651-656.

31. Zald DH, Cowan RL, Riccardi P, Baldwin RM, Ansari MS, Li R et al. Midbrain dopamine receptor availability is inversely associated with novelty-seeking traits in humans. $J$ Neurosci 2008; 28: 14372-14378.

32. Bunzeck N, Duzel E. Absolute coding of stimulus novelty in the human substantia nigra/ VTA. Neuron 2006; 51: 369-379.

33. Matsumoto M, Hikosaka $\mathrm{O}$. Two types of dopamine neuron distinctly convey positive and negative motivational signals. Nature 2009; 459: 837-841.

34. Salamone JD. The involvement of nucleus accumbens dopamine in appetitive and aversive motivation. Behav Brain Res 1994; 61: 117-133.

35. Kehagia AA, Murray GK, Robbins TW. Learning and cognitive flexibility: frontostriatal function and monoaminergic modulation. Curr Opin Neurobiol 2010; 20: 199-204.

36. Niv Y, Daw ND, Joel D, Dayan P. Tonic dopamine: opportunity costs and the control of response vigor. Psychopharmacology 2007; 191: 507-520.

37. Salamone JD, Correa M, Farrar AM, Nunes EJ, Pardo M. Dopamine, behavioral economics, and effort. Front Behav Neurosci 2009; 3: 13

38. Salamone JD, Correa M, Farrar A, Mingote SM. Effort-related functions of nucleus accumbens dopamine and associated forebrain circuits. Psychopharmacology 2007; 191: 461-482.

39. Floresco SB St, Onge JR, Ghods-Sharifi S, Winstanley CA. Cortico-limbic-striatal circuits subserving different forms of cost-benefit decision making. Cogn Affect Behav Neurosci 2008; 8: 375-389.

40. Satoh T, Nakai S, Sato T, Kimura M. Correlated coding of motivation and outcome of decision by dopamine neurons. J Neurosci 2003; 23: 9913-9923.

41. Adcock RA, Thangavel A, Whitfield-Gabrieli S, Knutson B, Gabrieli JD. Reward-motivated learning: mesolimbic activation precedes memory formation. Neuron 2006; 50: 507-517.

42. Boehler CN, Hopf JM, Krebs RM, Stoppel CM, Schoenfeld MA, Heinze HJ et al. Task-loaddependent activation of dopaminergic midbrain areas in the absence of reward. J Neurosci 2011; 31: 4955-4961.

43. Volkow ND, Wang GJ, Newcorn JH, Kollins SH, Wigal TL, Telang F et al. Motivation deficit in ADHD is associated with dysfunction of the dopamine reward pathway. Mol Psychiatry 2010; 16: 1147-1154.

44. Andersen ML, Perry JC, Tufik S. Acute cocaine effects in paradoxical sleep deprived male rats. Prog Neuropsychopharmacol Biol Psychiatry 2005; 29: 245-251.

45. Volkow ND, Wang GJ, Telang F, Fowler JS, Logan J, Wong C et al. Sleep deprivation decreases binding of [11C]raclopride to dopamine D2/D3 receptors in the human brain. J Neurosci 2008; 28: 8454-8461. 
46. Venkatraman V, Chuah YM, Huettel SA, Chee MW. Sleep deprivation elevates expectation of gains and attenuates response to losses following risky decisions. Sleep 2007; 30 : 603-609.

47. Stroop JR. Studies of interference in serial verbal reactions. J Exp Psychol 1935; 18 643-662.

48. Leung HC, Skudlarski $P$, Gatenby JC, Peterson BS, Gore JC. An event-related functiona MRI study of the stroop color word interference task. Cereb Cortex 2000; 10: 552-560.

49. Peterson BS, Kane MJ, Alexander GM, Lacadie C, Skudlarski P, Leung HC et al. An eventrelated functional MRI study comparing interference effects in the Simon and Stroop tasks. Brain Res Cogn Brain Res 2002; 13: 427-440.

50. Potenza MN, Leung HC, Blumberg HP, Peterson BS, Fulbright RK, Lacadie CM et al. An FMRI Stroop task study of ventromedial prefrontal cortical function in pathological gamblers. Am J Psychiatry 2003; 160: 1990-1994.

51. Blumberg HP, Leung HC, Skudlarski P, Lacadie CM, Fredericks CA, Harris BC et al. A functional magnetic resonance imaging study of bipolar disorder: state- and trait-related dysfunction in ventral prefrontal cortices. Arch Gen Psychiatry 2003; 60: 601-609.

52. Harrison BJ, Shaw M, Yucel M, Purcell R, Brewer WJ, Strother SC et al. Functiona connectivity during Stroop task performance. Neuroimage 2005; 24: 181-191.

53. Brewer JA, Worhunsky PD, Carroll KM, Rounsaville BJ, Potenza MN. Pretreatment brain activation during stroop task is associated with outcomes in cocaine-dependent patients. Biol Psychiatry 2008; 64: 998-1004.

54. Devito EE, Worhunsky PD, Carroll KM, Rounsaville BJ, Kober H, Potenza MN. A preliminary study of the neural effects of behavioral therapy for substance use disorders. Drug Alcohol Depend 2012; 122: 228-235.

55. Danielmeier C, Eichele T, Forstmann BU, Tittgemeyer M, Ullsperger M. Posterior media frontal cortex activity predicts post-error adaptations in task-related visual and motor areas. J Neurosci 2011; 31: 1780-1789.

56. Volkow ND, Fowler JS, Wang GJ, Swanson JM. Dopamine in drug abuse and addiction: results from imaging studies and treatment implications. Mol Psychiatry 2004; 9: 557-569.

57. Seamans JK, Yang CR. The principal features and mechanisms of dopamine modulation in the prefrontal cortex. Prog Neurobiol 2004; 74: 1-58.

58. Clatworthy PL, Lewis SJ, Brichard L, Hong YT, Izquierdo D, Clark L et al. Dopamine release in dissociable striatal subregions predicts the different effects of ora methylphenidate on reversal learning and spatial working memory. J Neurosci 2009; 29: 4690-4696.

59. Caparelli EC, Tomasi D, Arnold S, Chang L, Ernst T. k-Space based summary motion detection for functional magnetic resonance imaging. Neurolmage 2003; 20: 1411-1418.

60. Tomasi D, Caparelli EC, Chang L, Ernst T. fMRI-acoustic noise alters brain activation during working memory tasks. Neuroimage 2005; 27: 377-386.

61. Lee JH, Garwood M, Menon R, Adriany G, Andersen P, Truwit CL et al. High contrast and fast three-dimensional magnetic resonance imaging at high fields. Magn Reson Med 1995 34: 308-312.

62. Hennig J, Scheffler K. Hyperechoes. Magn Reson Med 2001; 46: 6-12.

63. Caparelli EC, Tomasi D. K-space spatial low-pass filters can increase signal loss artifacts in Echo-Planar Imaging. Biomed Signal Process Control 2008; 3: 107-114.

64. Ashburner J, Neelin P, Collins DL, Evans A, Friston K. Incorporating prior knowledge into image registration. Neuroimage 1997; 6: 344-352.

65. Friston KJ, Holmes AP, Worsley KJ, Poline JB, Frith CD, Frackowiak RS. Statistical parametric maps in functional imaging: a general approach. Hum Brain Mapp 1995; 2 : 189-210.

66. Schott BH, Minuzzi L, Krebs RM, Elmenhorst D, Lang M, Winz OH et al. Mesolimbic functional magnetic resonance imaging activations during reward anticipation correlate with reward-related ventral striatal dopamine release. J Neurosci 2008; 28: 14311-14319.

67. Urban NB, Slifstein M, Meda S, Xu X, Ayoub R, Medina O et al. Imaging human reward processing with positron emission tomography and functional magnetic resonance imaging. Psychopharmacology 2012; 221: 67-77.

68. Volkow ND, Fowler JS, Wang GJ, Dewey SL, Schlyer D, MacGregor R et al. Reproducibility of repeated measures of carbon-11-raclopride binding in the human brain. J Nucl Med 1993; 34: 609-613.

69. Kennerley SW, Walton ME, Behrens TE, Buckley MJ, Rushworth MF. Optimal decision making and the anterior cingulate cortex. Nat Neurosci 2006; 9: 940-947.

70. Boksem MA, Tops M. Mental fatigue: costs and benefits. Brain Res Rev 2008; 59 125-139.

71. Hester R, Garavan $\mathrm{H}$. Executive dysfunction in cocaine addiction: evidence for discordant frontal, cingulate, and cerebellar activity. J Neurosci 2004; 24: 11017-11022.

72. Forman SD, Dougherty GG, Casey BJ, Siegle GJ, Braver TS, Barch DM et al. Opiate addicts lack error-dependent activation of rostral anterior cingulate. Biol Psychiatry 2004 55: 531-537.
73. Kaufman JN, Ross TJ, Stein EA, Garavan H. Cingulate hypoactivity in cocaine users during a GO-NOGO task as revealed by event-related functional magnetic resonance imaging J Neurosci 2003; 23: 7839-7843.

74. Hester R, Nestor L, Garavan H. Impaired error awareness and anterior cingulate cortex hypoactivity in chronic cannabis users. Neuropsychopharmacology 2009; 34: 2450-2458.

75. Yücel M, Lubman DI, Harrison BJ, Fornito A, Allen NB, Wellard RM et al. A combined spectroscopic and functional MRI investigation of the dorsal anterior cingulate region in opiate addiction. Mol Psychiatry 2007; 12: 611 691-702.

76. Barnden LR, Crouch B, Kwiatek R, Burnet R, Mernone A, Chryssidis S et al. A brain MR study of chronic fatigue syndrome: evidence of brainstem dysfunction and altered homeostasis. NMR Biomed 2011; 24: 1302-1312.

77. Bruno RL, Creange SJ, Frick NM. Parallels between post-polio fatigue and chronic fatigue syndrome: a common pathophysiology? Am J Med 1998; 105: 66S-73S

78. Davis JM, Bailey SP. Possible mechanisms of central nervous system fatigue during exercise. Med Sci Sports Exerc 1997; 29: 45-57.

79. Foley TE, Fleshner M. Neuroplasticity of dopamine circuits after exercise: implications for central fatigue. Neuromolecular Med 2008; 10: 67-80.

80. Bromberg-Martin ES, Matsumoto M, Hikosaka O. Dopamine in motivational control: rewarding, aversive, and alerting. Neuron 2010; 68: 815-834.

81. Chevrier A, Schachar RJ. Error detection in the stop signal task. Neuroimage 2010; 53 664-673.

82. Milham MP, Banich MT, Claus ED, Cohen NJ. Practice-related effects demonstrate complementary roles of anterior cingulate and prefrontal cortices in attentional control. Neuroimage 2003; 18: 483-493.

83. Volkow ND, Fowler JS, Wang GJ, Telang F, Logan J, Wong C et al. Methylphenidate decreased the amount of glucose needed by the brain to perform a cognitive task. PLoS ONE 2008; 3: e2017

84. Abe K, Takanashi M, Yanagihara T, Sakoda S. Pergolide mesilate may improve fatigue in patients with Parkinson's disease. Behav Neurol 2001; 13: 117-121.

85. Caldwell JA, Caldwell JL. An in-flight investigation of the efficacy of dextroamphetamine for sustaining helicopter pilot performance. Aviat Space Environ Med 1997; 68 1073-1080.

86. Li CS, Morgan PT, Matuskey D, Abdelghany O, Luo X, Chang JL et al. Biological markers of the effects of intravenous methylphenidate on improving inhibitory control in cocainedependent patients. Proc Natl Acad Sci USA 2010; 107: 14455-14459.

87. Goldstein RZ, Woicik PA, Maloney T, Tomasi D, Alia-Klein N, Shan J et al. Ora methylphenidate normalizes cingulate activity in cocaine addiction during a salient cognitive task. Proc Natl Acad Sci USA 2010; 107: 16667-16672.

88. Hannestad J, Gallezot JD, Planeta-Wilson B, Lin SF, Williams WA, van Dyck CH et a. Clinically relevant doses of methylphenidate significantly occupy norepinephrine transporters in humans in vivo. Biol Psychiatry 2010; 68: 854-860.

89. Heimovics SA, Salvante KG, Sockman KW, Riters LV. Individual differences in the motivation to communicate relate to levels of midbrain and striatal catecholamine markers in male European starlings. Horm Behav 2011; 60: 529-539.

90. Schoenmakers TM, de Bruin M, Lux IF, Goertz AG, Van Kerkhof DH, Wiers RW. Clinical effectiveness of attentional bias modification training in abstinent alcoholic patients. Drug Alcohol Depend 2010; 109: 30-36.

91. Muraven M. Practicing self-control lowers the risk of smoking lapse. Psychol Addict Behav 2010; 24: 446-452.

92. Wilkinson G. The Wide-Range Achievement Test 3-Administration Manual. Wide Range Inc.: Wilmington, DE, 1993.

93. Wechsler D. Wechsler Abbreviated Scale of Intelligence. Psychological Corporation: San Antonio, TX, 1999

94. Beck AT, Steer RA, Brown GK. Beck Depression Inventory Manual. 2nd edn The Psychological Corporation: San Antonio, 1996.

95. Kampman KM, Volpicelli JR, McGinnis DE, Alterman Al, Weinrieb RM, D'Angelo L et al. Reliability and validity of the Cocaine Selective Severity Assessment. Addict Behav 1998 23: 449-461.

96. Tiffany ST, Singleton E, Haertzen CA, Henningfield JE. The development of a cocaine craving questionnaire. Drug Alcohol Depend 1993; 34: 19-28.

Translational Psychiatry is an open-access journal published by Nature Publishing Group. This work is licensed under the Creative Commons Attribution-NonCommercial-No Derivative Works 3.0 Unported License. To view a copy of this license, visit http://creativecommons.org/licenses/by-nc-nd/3.0/ 